\title{
Significance of fluctuations in serum sodium levels following aneurysmal subarachnoid hemorrhage: an exploratory analysis
}

\author{
Matthew E. Eagles, MD,1 Michael K. Tso, MD, ${ }^{1}$ and R. Loch Macdonald, MD, PhD ${ }^{2,3}$ \\ 1Section of Neurosurgery, Department of Clinical Neurosciences, University of Calgary, Alberta; ${ }^{2}$ Division of Neurosurgery and \\ Li Ka Shing Knowledge Institute, St. Michael's Hospital, Toronto; and ${ }^{3}$ Institute of Medical Science, University of Toronto, Ontario, \\ Canada
}

OBJECTIVE Fluctuations in patient serum sodium levels are common after aneurysmal subarachnoid hemorrhage (aSAH), but their effect on patient outcome is not well described in the literature. The goal of this work was to better characterize the relationship between fluctuations in serum sodium levels, outcome, and the development of delayed cerebral ischemia (DCl) after aSAH.

METHODS The authors performed a post hoc analysis of data from the Clazosentan to Overcome Neurological Ischemia and Infarction Occurring After Subarachnoid Hemorrhage (CONSCIOUS-1) trial. Patients had their serum sodium values recorded daily for 14 days post-aSAH. Average and average absolute daily differences in sodium levels were calculated for each patient based on 3 reference points: admission sodium levels, a normal sodium level (defined as 140 $\mathrm{mmol} / \mathrm{L}$ ), and the previous day's sodium level. These variables were also calculated for the classic "vasospasm window" (days 3-12) post-aSAH. A stepwise logistic regression model, locally weighted scatterplot smoothing curves, and receiver operator characteristic curve analysis were used to evaluate the relationship between alterations in serum sodium levels and clinical outcome or the development of $\mathrm{DCl}$ after aSAH. Poor outcome was defined as a modified Rankin Scale (mRS) score of $>2$ at 3 months.

RESULTS The average daily difference in sodium values from baseline $(p<0.001)$, average daily difference from a normal sodium level $(p<0.001)$, average absolute daily difference from a normal sodium level $(p=0.015)$, and average absolute daily difference from the previous day's sodium level $(p=0.017)$ were significant predictors of poor outcome in a stepwise multivariate regression model. There was a trend toward significance for average absolute daily difference from admission sodium levels during the vasospasm window as an independent predictor of $\mathrm{DCl}(\mathrm{p}=0.052)$. There was no difference in the predictive capacity for $\mathrm{DCl}$ when sodium fluctuations from post-aSAH days 1-14 were compared with those from the classic vasospasm window (days 3-12).

CONCLUSIONS Fluctuations in serum sodium levels may play a role in clinical outcome and the development of DCl after aSAH. The timing of these fluctuations appears to have no significant effect on the development of $\mathrm{DCl}$.

https://thejns.org/doi/abs/10.3171/2018.3.JNS173068

KEYWORDS subarachnoid hemorrhage; sodium; delayed cerebral ischemia; vascular disorders

$\mathrm{A}$ NEURYSMAL subarachnoid hemorrhage (aSAH) is a devastating neurological condition with a worldwide incidence of approximately 600,000 cases per year. ${ }^{5}$ Outcome after aSAH is dependent on early factors such as patient age, the patient's neurological condition on presentation, and the amount of blood visible on the initial CT scan. ${ }^{13,18}$ It is also dependent on complica- tions throughout the course of hospital stay, such as aneurysm rebleeding and the development of delayed cerebral ischemia (DCI) ${ }^{7,8,22}$

Deviations in serum sodium levels are common after aSAH, and dysnatremia has been reported in more than half of patients in select studies..$^{11,15,21}$ Nonetheless, the relationship between these sodium alterations and outcome

ABBREVIATIONS aSAH = aneurysmal subarachnoid hemorrhage; CONSCIOUS-1 = Clazosentan to Overcome Neurological Ischemia and Infarction Occurring After Subarachnoid Hemorrhage; $\mathrm{DCl}=$ delayed cerebral ischemia; LOWESS = locally weighted scatterplot smoothing; mRS = modified Rankin Scale; WFNS = World Federation of Neurosurgical Societies.

SUBMITTED December 9, 2017. ACCEPTED March 12, 2018.

INCLUDE WHEN CITING Published online August 17, 2018; DOI: 10.3171/2018.3.JNS173068. 
after aSAH remains unclear. Although guidelines have been developed to address the issue of hyponatremia in patients who experience aSAH,${ }^{17}$ there are relatively few studies that have described a relationship between hyponatremia and poor outcome. ${ }^{20,23}$ Conversely, a recent systematic review by Mapa et al. ${ }^{11}$ noted that hyponatremia might be associated with certain poor outcomes, such as DCI and duration of hospital stay, but it did not find evidence to suggest that it affected mortality. Still, it did note that there was a need for stronger evidence in the form of larger prospective studies. Other work has suggested that it is not hyponatremia, but fluctuations in serum sodium or the development of hypernatremia that is detrimental to patient outcome. ${ }^{1,2}$

The current study aimed to assess whether the development of dysnatremia affected the outcome of patients enrolled in the Clazosentan to Overcome Neurological Ischemia and Infarction Occurring After Subarachnoid Hemorrhage (CONSCIOUS-1) trial. ${ }^{9}$ We also sought to assess whether these fluctuations were predictive of DCI or whether the timing of sodium deviations had any impact on the risk of developing DCI.

\section{Methods \\ Patient Population}

This study was a post hoc analysis of the 413 patients enrolled in the CONSCIOUS-1 trial (clinical trial registration no.: NCT00111085 [clinicaltrials.gov]) from January 2005 to March 2006. The methods and results for this trial have been previously published. ${ }^{9}$ This project was approved by the institutional review board at St. Michael's Hospital (Toronto, Ontario).

\section{Data}

Patients had their serum sodium levels checked daily from hospital admission to post-aSAH day 14. Average daily differences from admission sodium levels, normal sodium levels (defined as $140 \mathrm{mmol} / \mathrm{L}$ ), and the previous day's sodium levels were calculated for every patient. The average absolute daily differences from the same reference points were also calculated. Lastly, these variables were also computed for the classic "vasospasm window," which was defined as post-aSAH days 3-12.25

The primary outcome measure was poor patient outcome, which was defined as a modified Rankin Scale (mRS) score of greater than 2 at 3 months. ${ }^{26}$ Secondary outcome was the development of DCI. This was defined as a decrease of 2 points on the Glasgow Coma Scale or an increase of 2 points on the abbreviated National Institutes of Health Stroke Scale score that lasted for at least 2 hours and was associated with angiographic vasospasm. DCI was also defined by a new infarction on neuroimaging not attributable to any other cause, or, for patients unable to tolerate a neurological examination, clinical signs of DCI (new neurological deficit or unexplained fever) in association with angiographic vasospasm. ${ }^{9}$

\section{Statistical Analysis}

Baseline characteristics, including clinical status and imaging variables, of the study group were compared us- ing univariate logistic regression to determine their effect on patient outcome and the development of DCI. World Federation of Neurosurgical Societies (WFNS) grades were dichotomized as good (I-III) and poor (IV and V). Hypertonic saline and mannitol administration were analyzed as dichotomous variables, while the Fisher scale was analyzed as a categorical variable.

A stepwise multivariate regression model was created to assess for independent predictors of poor outcome and DCI. All characteristics with $\mathrm{p}<0.10$ on univariate analysis were included in the multivariate models (Table 1). To avoid collinearity, the multivariate analysis was repeated for each measure of sodium fluctuation that met criteria for inclusion into the model. Locally weighted scatterplot smoothing (LOWESS) curves were used to visualize whether the aforementioned measures of sodium fluctuation affected patient outcome or the development of DCI. Receiver operating characteristic curve analysis was used to assess for differences in the predictive capacity for DCI between absolute average daily change in sodium values during the classic vasospasm window (post-aSAH days 3-12) and days 1-14 post-aSAH.

The significance level was set at $p<0.05$. Statistical analysis was carried out using Stata software (version 15.1, StataCorp).

\section{Results}

Of the 413 patients in the CONSCIOUS- 1 data set, 130 $(31 \%)$ had a poor outcome (mRS score $>2$ ), and $78(19 \%)$ developed DCI. Baseline characteristics of the study cohort and the results of univariate logistic regression are outlined in Table 1. Poor WFNS grades (IV or V) (OR $5.21,95 \%$ CI 3.22-8.42), age (OR 1.03, 95\% CI 1.01-1.05), female sex (OR 1.78, 95\% CI 1.10-2.90), a history of hypertension (OR 2.27, 95\% CI 1.47-3.47), mannitol use (OR $2.48,95 \%$ CI 1.62-3.80), and the development of DCI (OR $5.69,95 \%$ CI 3.36-9.64) were significantly associated with poor outcome on univariate analysis. Fisher grade $2 \mathrm{aSAH}$ on initial head CT scanning was protective against poor outcome (OR 0.40, 95\% CI 0.22-0.73). There was a trend toward poor outcome for patients who had higher baseline sodium values on admission, but this did not reach statistical significance (OR 1.05, 95\% CI 1.00-1.10). Hypertonic saline administration and a diagnosis of diabetes or hyperglycemia also met inclusion criteria for the multivariate model.

Univariate regression results of all sodium fluctuation measures tested are outlined in Table 2. Average daily difference from admission sodium levels (OR 1.09, 95\% CI 1.04-1.14), average absolute daily difference from admission sodium levels (OR 1.13, 95\% CI 1.05-1.22), average daily difference from a normal sodium level (OR 1.18, 95\% CI 1.12-1.25), average absolute daily difference from a normal sodium level (OR 1.19, 95\% CI 1.10-1.30), and average absolute daily difference from the previous day's sodium level (OR 1.29, 95\% CI 1.10-1.50) were found to be associated with poor outcome.

In a multivariate model combining all of the variables with $\mathrm{p}<0.10$ on univariate testing, the following sodium fluctuation measures were found to be independent pre- 
TABLE 1. Baseline characteristics of the study cohort

\begin{tabular}{|c|c|c|c|c|}
\hline & Total $(n=413)$ & mRS Score $\leq 2(n=283)$ & mRS Score >2 (n=130) & OR (95\% Cl, p value) \\
\hline Mean age, yrs (SD)* & $50.99(10.77)$ & $50.08(10.57)$ & $52.97(10.99)$ & $1.03(1.01-1.05,0.012)$ \\
\hline Female sex* & $292(71 \%)$ & $190(65 \%)$ & $102(35 \%)$ & $1.78(1.10-2.90,0.020)$ \\
\hline History of hypertension* & $172(42 \%)$ & $100(58 \%)$ & $72(42 \%)$ & $2.27(1.49-3.47,<0.01)$ \\
\hline Smoking history & $217(53 \%)$ & $155(71 \%)$ & $62(29 \%)$ & $0.84(0.55-1.29,0.42)$ \\
\hline Poor WFNS grade (IV or V) ${ }^{*}$ & $100(24 \%)$ & $40(40 \%)$ & $60(60 \%)$ & $5.21(3.22-8.42,<0.01)$ \\
\hline $\mathrm{DCl}^{*}$ & $78(19 \%)$ & $28(36 \%)$ & $50(64 \%)$ & $5.69(3.36-9.64,<0.001)$ \\
\hline \multicolumn{5}{|l|}{ Fisher grade* } \\
\hline 1 & $4(1 \%)$ & $4(100 \%)$ & $0(0 \%)$ & NA \\
\hline 2 & $85(21 \%)$ & $69(81 \%)$ & $16(19 \%)$ & $0.40(0.22-0.73,0.003)$ \\
\hline 3 & $35(8 \%)$ & $27(77 \%)$ & $8(23 \%)$ & $0.51(0.22-1.17,0.113)$ \\
\hline 4 & $284(69 \%)$ & $180(63 \%)$ & $104(37 \%)$ & NA \\
\hline Given hypertonic saline* & $53(13 \%)$ & $31(58 \%)$ & $22(42 \%)$ & $1.66(0.92-2.99,0.094)$ \\
\hline Given mannitol* $^{*}$ & $191(46 \%)$ & $111(58 \%)$ & $80(42 \%)$ & $2.48(1.62-3.80,<0.001)$ \\
\hline Diabetes or hyperglycemia* & $53(13 \%)$ & $31(58 \%)$ & $22(42 \%)$ & $1.66(0.92-2.99,0.094)$ \\
\hline Mean initial serum sodium value, $\mathrm{mmol} / \mathrm{L}(\mathrm{SD})^{*}$ & $139.6(4.16)$ & $139.3(3.95)$ & $140.2(4.54)$ & $1.05(1.00-1.10,0.06)$ \\
\hline
\end{tabular}

$\mathrm{NA}=$ not assessed due to perfect prediction or collinearity.

* Variable included in multivariate model.

dictors of poor outcome: average daily difference in sodium values from baseline (OR 1.13, 95\% CI 1.06-1.20), average daily difference from a normal sodium level (OR $1.17,95 \%$ CI 1.08-1.26), average absolute daily difference from a normal sodium level (OR 1.14, 95\% CI 1.03-1.26), and average absolute daily difference from the previous day's sodium level (OR 1.24, 95\% CI 1.04-1.48) (Table 2). LOWESS curves demonstrated an increased proportion of patients who had a poor outcome with increasing average daily differences from baseline sodium values (Fig. 1).

No measures of sodium fluctuation were found to be independent predictors of DCI on multivariate analysis after accounting for poor WFNS grade (IV or V), initial Fisher grade, hypertonic saline use, and mannitol use. However, there was a strong trend toward significance for average absolute daily difference from baseline during the vasospasm window $(p=0.052)$. LOWESS curves showed that a greater proportion of patients suffered DCI as sodium values moved in both the positive and negative directions from baseline values (Fig. 2). There was no significant difference in the area under the receiver operating characteristic curve for absolute average daily change in sodium levels from baseline during the classic vasospasm window (days 3-12) compared with post-aSAH days 1-14 (0.605 vs $0.607, \mathrm{p}=0.86$ ).

\section{Discussion}

\section{Effect of Sodium Fluctuations on Outcome After aSAH}

Our analysis demonstrated that fluctuations in serum sodium values may play a role in both outcome and development of DCI in patients who have experienced aSAH. LOWESS curves suggested that an increased proportion of patients have poor outcome and DCI with greater fluctuations in their sodium levels; however, for patient outcome this was only true as sodium levels increased from their baseline values on admission, whereas for DCI it was true regardless of the direction the sodium trended. Lastly, there did not appear to be any difference in the predictive value of fluctuations in serum sodium values as a predictor for DCI during the vasospasm period (days 3-12) compared with the entirety of the study period (days 1-14).

Classically, hyponatremia after aSAH is thought to be due to one of 2 principal causes: syndrome of inappro-

TABLE 2. Effect of sodium fluctuation measures on outcome after aSAH

\begin{tabular}{lcc}
\hline \multicolumn{1}{c}{ Sodium Fluctuation Measure } & Univariate OR $(95 \% \mathrm{Cl}, \mathrm{p}$ value $)$ & Multivariate OR $(95 \% \mathrm{Cl}, \mathrm{p}$ value $)$ \\
\hline Average daily difference from admission sodium level & $1.09(1.04-1.14,<0.001)$ & $1.13(1.06-1.20,<0.001)$ \\
\hline Average absolute daily difference from admission sodium level & $1.13(1.05-1.22,0.001)$ & $1.08(1.00-1.07,0.062)$ \\
\hline Average daily difference from normal sodium level $(140 \mathrm{mmol} / \mathrm{L})$ & $1.18(1.12-1.25,<0.001)$ & $1.17(1.08-1.26,<0.001)$ \\
\hline Average absolute daily difference from normal sodium level $(140 \mathrm{mmol} / \mathrm{L})$ & $1.19(1.10-1.30,<0.001)$ & $1.14(1.03-1.26,0.015)$ \\
\hline Average daily difference from previous day's sodium level & $1.22(0.83-1.80,0.303)$ & - \\
\hline Average absolute daily difference from previous day's sodium level & $1.29(1.10-1.50,0.002)$ & $1.24(1.04-1.48,0.017)$ \\
\hline
\end{tabular}

$-=$ did not meet criteria for multivariate model. 


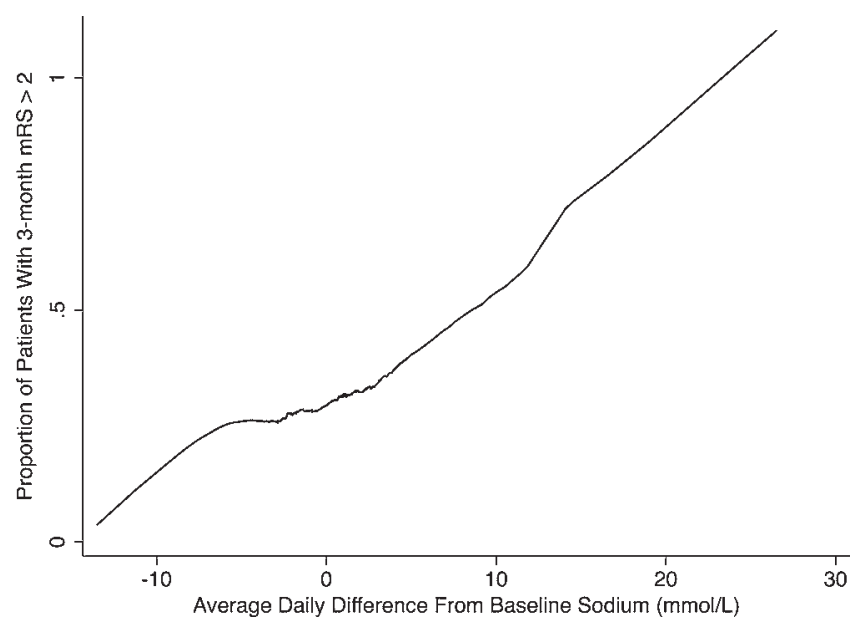

FIG. 1. LOWESS curve demonstrating the relationship between the proportion of patients who had a poor outcome (mRS score $>2$ at 3 months) and their average daily change in serum sodium ( $\mathrm{mmol} / \mathrm{L}$ ) from their baseline levels on admission.

priate secretion of antidiuretic hormone and cerebral salt wasting. ${ }^{4,14,16}$ Hypernatremia following aSAH is also common and has been associated with poor prognosis in previous studies. ${ }^{6,15,24}$ However, in their article published in 2006, Wartenberg et al. correctly pointed out that hypernatremia following aSAH is likely a result of hypertonic therapy for the treatment of intracranial hypertension, thus confounding the impact of hypernatremia on outcome. ${ }^{24}$ Nonetheless, 1 study found that the presence of early hypernatremia (within 56 hours of ictus) was correlated with poor WFNS grade and poor outcome at 12 months, indicating that hypernatremia may be related to early brain injury following aSAH. ${ }^{2}$ Our work found a similar trend, but increasing admission sodium values did not quite reach statistical significance as a univariate predictor of poor outcome after aSAH.

The effect of hyponatremia on outcome after aSAH remains unclear. Many studies found no relationship between low serum sodium and outcome., ${ }^{1,15,24}$ A recent systematic review found conflicting evidence for the effect of hyponatremia on outcome but did note that there was an association between hyponatremia and duration of ICU stay and DCI. ${ }^{11}$ Our work suggests that sodium fluctuations are a significant predictor of a poor outcome. However, our graphic analysis suggests that, while decreasing serum sodium values may play a role in the development of DCI after aSAH, the proportion of patients who have a poor outcome decreased as sodium levels trended in the negative direction.

Hyponatremia after aSAH has been associated with poor clinical grade on admission and clot volume. ${ }^{10}$ As such, it may be that the severity of the bleed in aSAH impairs the pituitary gland's ability to regulate sodium homeostasis, leading to the development of dysnatremia. Our work relied heavily on the use of graphic analysis to tease out the effect of sodium fluctuations on clinical outcome, particularly decreases in serum sodium. Our reason for this was, as mentioned previously, hypernatremia after

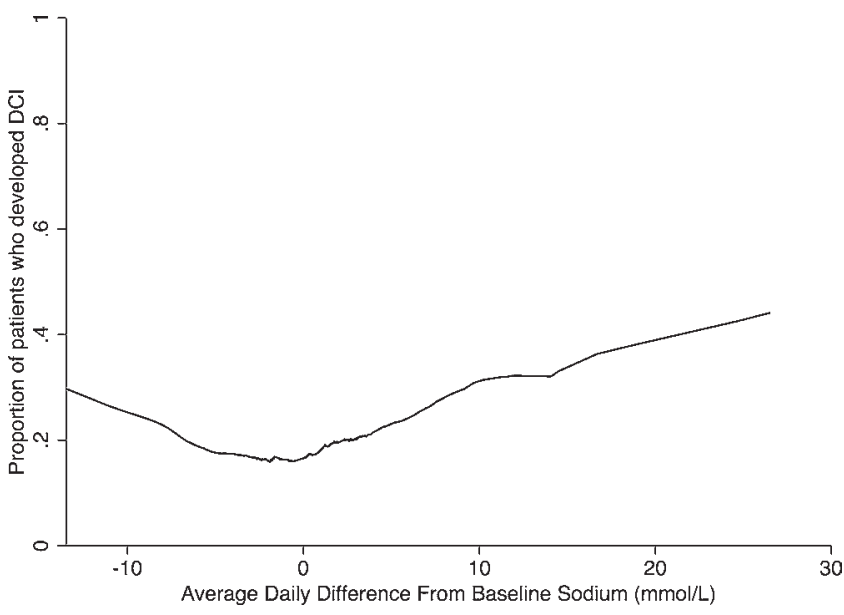

FIG. 2. LOWESS curve demonstrating the relationship between the proportion of patients who developed $\mathrm{DCl}$ and their average daily change in serum sodium ( $\mathrm{mmol} / \mathrm{L}$ ) from their baseline levels on admission.

aSAH is commonly induced by hypertonic therapy to treat patients with intracranial hypertension, and these patients are more likely to have a poor outcome, regardless of their sodium level. ${ }^{24}$

Fluctuations in serum sodium have previously been associated with poor outcome in surgical ICU patients and in aSAH. ${ }^{1,19}$ However, these studies considered only absolute deviation in serum sodium and did not demonstrate whether the direction of sodium fluctuations impacted outcomes. Furthermore, the paper by Bales et al. considered only patient outcome at discharge, as opposed to clinical outcome at 3 months, a time when patients have had a chance to undergo more recovery and rehabilitation. ${ }^{1} \mathrm{We}$ feel that our paper expands upon these works, as it demonstrates the association between both increasing serum sodium values and poor outcome at 3 months, with the inclusion of hypertonic therapy in our multivariate model. However, it is notable that decreasing serum sodium values were not associated with poor clinical outcome in our study.

\section{Effect of Sodium Fluctuations on Risk of DCI After aSAH}

The association between sodium values and DCI is more clearly established. The literature contains several studies that have shown that hyponatremia is associated with an increased risk of DCI. 3,10,12,23,27 Our graphic analysis suggests that there is an increased risk of DCI when sodium levels fluctuate in both the positive and negative directions from baseline values on admission. However, multivariate analysis did not show sodium fluctuations to be an independent predictor of DCI, although there was a strong trend toward significance for average absolute daily difference from baseline during the vasospasm window. Interestingly, only one of the studies evaluated the timing of dysnatremia on the development of DCI. While that work showed that late-onset (defined as post-SAH day 3) hyponatremia was more predictive of DCI, ${ }^{27}$ our data did not support this conclusion.

There are a few strengths and limitations to our study 
that deserve mention. First, our data were prospectively collected. All patients were part of a large randomized control trial, and the data set for the CONSCIOUS-1 trial was heavily audited. Furthermore, our outcome data were collected at 3 months, as opposed to hospital discharge, thus giving a better picture of the patients' long-term level of function. In terms of limitations, our study was an exploratory analysis, and the data were not collected for the purposes of comparing serum sodium values. As mentioned previously, the effect of hypertonic therapy for the treatment of intracranial hypertension remains unclear and may significantly confound the results for patients with hypernatremia. Although we were able to evaluate which patients received hypertonic saline, the exact timing and dose of hypertonic therapy was not readily available. Furthermore, the goal serum sodium for the CONSCIOUS-1 trial was not accessible for our purposes.

Future work should attempt to better characterize the effect of serum sodium changes in aSAH patients, while better accounting for those who receive hypertonic saline therapy for elevated intracranial pressure. A prospective trial, created for the sole purpose of evaluating the effect of dysnatremia on outcome and DCI in aSAH patients, would also be worth pursuing.

\section{Conclusions}

In our retrospective analysis of aSAH patients enrolled in a clinical trial, we found that positive serum sodium fluctuations were associated with poor outcome. Graphically, there is an increased risk of DCI with negative and positive serum sodium deviations. Sodium fluctuations within the classic vasospasm period do not appear to confer an increased risk of DCI compared with sodium fluctuations during the entire 14-day period. More work is needed to elucidate the relationship between hypertonic therapy, sodium fluctuations, and how the combination of these factors affect patient outcome following aSAH.

\section{References}

1. Bales J, Cho S, Tran TK, Korab GA, Khandelwal N, Spiekerman CF, et al: The effect of hyponatremia and sodium variability on outcomes in adults with aneurysmal subarachnoid hemorrhage. World Neurosurg 96:340-349, 2016

2. Beseoglu K, Etminan N, Steiger HJ, Hänggi D: The relation of early hypernatremia with clinical outcome in patients suffering from aneurysmal subarachnoid hemorrhage. Clin Neurol Neurosurg 123:164-168, 2014

3. Chandy D, Sy R, Aronow WS, Lee WN, Maguire G, Murali R: Hyponatremia and cerebrovascular spasm in aneurysmal subarachnoid hemorrhage. Neurol India 54:273-275, 2006

4. Diringer MN, Bleck TP, Claude Hemphill J III, Menon D, Shutter L, Vespa P, et al: Critical care management of patients following aneurysmal subarachnoid hemorrhage: recommendations from the Neurocritical Care Society's Multidisciplinary Consensus Conference. Neurocrit Care 15:211-240, 2011

5. Feigin VL, Lawes CMM, Bennett DA, Barker-Collo SL, Parag V: Worldwide stroke incidence and early case fatality reported in 56 population-based studies: a systematic review. Lancet Neurol 8:355-369, 2009

6. Fisher LA, Ko N, Miss J, Tung PP, Kopelnik A, Banki NM, et al: Hypernatremia predicts adverse cardiovascular and neurological outcomes after SAH. Neurocrit Care 5:180185,2006

7. Kassell NF, Sasaki T, Colohan ART, Nazar G: Cerebral vasospasm following aneurysmal subarachnoid hemorrhage. Stroke 16:562-572, 1985

8. Macdonald RL: Delayed neurological deterioration after subarachnoid haemorrhage. Nat Rev Neurol 10:44-58, 2014

9. Macdonald RL, Kassell NF, Mayer S, Ruefenacht D, Schmiedek P, Weidauer S, et al: Clazosentan to overcome neurological ischemia and infarction occurring after subarachnoid hemorrhage (CONSCIOUS-1): randomized, double-blind, placebo-controlled phase 2 dose-finding trial. Stroke 39:3015-3021, 2008

10. Maimaitili A, Maimaitili M, Rexidan A, Lu J, Ajimu K, Cheng X, et al: Pituitary hormone level changes and hypxonatremia in aneurysmal subarachnoid hemorrhage. Exp Ther Med 5:1657-1662, 2013

11. Mapa B, Taylor BES, Appelboom G, Bruce EM, Claassen J, Connolly ES Jr: Impact of hyponatremia on morbidity, mortality, and complications after aneurysmal subarachnoid hemorrhage: a systematic review. World Neurosurg 85:305314, 2016

12. Nakagawa I, Kurokawa S, Nakase H: Hyponatremia is predictable in patients with aneurysmal subarachnoid hemorrhage-clinical significance of serum atrial natriuretic peptide. Acta Neurochir (Wien) 152:2147-2152, 2010

13. Park J, Woo H, Kang DH, Kim Y: Critical age affecting 1 -year functional outcome in elderly patients aged $\geq 70$ years with aneurysmal subarachnoid hemorrhage. Acta Neurochir (Wien) 156:1655-1661, 2014

14. Peters JP, Welt LG, Sims EAH, Orloff J, Needham J: A saltwasting syndrome associated with cerebral disease. Trans Assoc Am Physicians 63:57-64, 1950

15. Qureshi AI, Suri MFK, Sung GY, Straw RN, Yahia AM, Saad M, et al: Prognostic significance of hypernatremia and hyponatremia among patients with aneurysmal subarachnoid hemorrhage. Neurosurgery 50:749-756, 2002

16. Rabinstein AA, Wijdicks EFM: Hyponatremia in critically ill neurological patients. Neurologist 9:290-300, 2003

17. Rahman M, Friedman WA: Hyponatremia in neurosurgical patients: clinical guidelines development. Neurosurgery 65:925-936, 2009

18. Rosengart AJ, Schultheiss KE, Tolentino J, Macdonald RL: Prognostic factors for outcome in patients with aneurysmal subarachnoid hemorrhage. Stroke 38:2315-2321, 2007

19. Sakr Y, Rother S, Ferreira AM, Ewald C, Dünisch P, Riedemmann N, et al: Fluctuations in serum sodium level are associated with an increased risk of death in surgical ICU patients. Crit Care Med 41:133-142, 2013

20. Saramma P, Menon RG, Srivastava A, Sarma PS: Hyponatremia after aneurysmal subarachnoid hemorrhage: implications and outcomes. J Neurosci Rural Pract 4:24-28, 2013

21. Sherlock M, O’Sullivan E, Agha A, Behan LA, Rawluk D, Brennan P, et al: The incidence and pathophysiology of hyponatraemia after subarachnoid haemorrhage. Clin Endocrinol (Oxf) 64:250-254, 2006

22. Starke RM, Connolly ES Jr: Rebleeding after aneurysmal subarachnoid hemorrhage. Neurocrit Care 15:241-246, 2011

23. Vrsajkov V, Javanović G, Stanisavljević S, Uvelin A, Vrsajkov JP: Clinical and predictive significance of hyponatremia after aneurysmal subarachnoid hemorrhage. Balkan Med J 29:243-246, 2012

24. Wartenberg KE, Schmidt JM, Claassen J, Temes RE, Frontera JA, Ostapkovich N, et al: Impact of medical complications on outcome after subarachnoid hemorrhage. Crit Care Med 34:617-624, 2006

25. Weir B, Grace M, Hansen J, Rothberg C: Time course of vasospasm in man. J Neurosurg 48:173-178, 1978 
26. Weisscher N, Vermeulen M, Roos YB, de Haan RJ: What should be defined as good outcome in stroke trials; a modified Rankin score of 0-1 or 0-2? J Neurol 255:867-874, 2008

27. Zheng B, Qiu Y, Jin H, Wang L, Chen X, Shi C, et al: A predictive value of hyponatremia for poor outcome and cerebral infarction in high-grade aneurysmal subarachnoid haemorrhage patients. J Neurol Neurosurg Psychiatry 82:213-217, 2011

\section{Disclosures}

Dr. Macdonald reports ownership of Edge Therapeutics, Inc. and receiving non-study-related clinical or research effort support from the Brain Aneurysm Foundation, Heart and Stroke Foundation of Canada, Canadian Institutes for Health Research, and Physicians Services Incorporated Foundation.

\section{Author Contributions}

Conception and design: Eagles, Tso. Acquisition of data: Macdonald. Analysis and interpretation of data: Eagles, Tso. Critically revising the article: Tso, Macdonald. Reviewed submitted version of manuscript: Tso, Macdonald. Statistical analysis: Eagles, Tso. Study supervision: Macdonald.

\section{Supplemental Information}

Previous Presentations

Portions of this work were presented in abstract form as an oral presentation at the 2017 Congress of Neurological Surgeons Annual Meeting, Boston, MA, October 9, 2017.

\section{Correspondence}

Matthew E. Eagles: University of Calgary, AB, Canada. matthew. eagles@ucalgary.ca. 